\title{
Polariton traps in semiconductor microcavities
}

\author{
Jeremy J. Baumberg ${ }^{\mathrm{a}, *}$, Pavlos G. Savvidis ${ }^{\mathrm{a}}$, Pavlos. G. Lagoudakis ${ }^{\mathrm{a}}$, M.D. Martin ${ }^{\mathrm{a}}$, \\ David Whittaker ${ }^{\mathrm{a}}, \mathrm{R}$. Butte ${ }^{\mathrm{b}}$, Maurice Skolnick ${ }^{\mathrm{b}}$, John Roberts $^{\mathrm{c}}$ \\ a Department of Physics \& Astronomy, University of Southampton, SO17 1BJ, UK \\ ${ }^{\mathrm{b}}$ Department of Physics, University of Sheffield, Sheffield S3 7RH, UK \\ ${ }^{\mathrm{c}}$ Department of Electronic and Electrical Engineering, University of Sheffield, Sheffield, S1 3JD, UK
}

\begin{abstract}
The strong coupling of excitons and photons inside a semiconductor microcavity forms a trap in $k$-space for the coupled polaritons. This trap produces new behaviour from the strong parametric scattering of polaritons on the walls of the trap, from condensation of polaritons at the bottom of the trap and from the bottleneck in relaxation at the neck of the trap. We predict the operation of polariton lasers pumped non-resonantly, either electrically or optically, based on efficient scattering of polaritons into the trap. (c) 2002 Elsevier Science B.V. All rights reserved.
\end{abstract}

Keywords: Stimulation; Polaritons; Microcavities; Amplification; Condensates

Since the first measurement of the angular dispersion of semiconductor microcavities [1], it has become clear that the in-plane wave vector of the mixed exciton-cavity photons is extremely important for their linear and nonlinear properties. The strong coupling of the highly dispersive cavity photon mode with the rather flat quadratic exciton dispersion, leads to new upper and lower polariton dispersions which are severely distorted, split by the Rabi frequency, $\Omega$. The dominant new feature is the deep minimum in the energy-momentum dispersion of the lower polariton, which has a depth $\Omega / 2$, and a width, $\Delta k=\sqrt{12 \varepsilon \omega \Omega} / \hbar c$, where $\varepsilon$ is the dielectric constant (Fig. 1). This forms a trap for polaritons, similar to the real-space traps developed for atoms, but existing in $k$-space. In the same way that evaporative cooling

\footnotetext{
* Corresponding author. Tel.: +44-23-8059-3911; fax: +44-238059-3910.

E-mail address: j.j.baumberg@soton.ac.uk (J.J. Baumberg).
}

can be used to allow a cold gas of atoms to Bose-condense into macroscopic quantum state, the excitons are able to pair scatter in such a way as to produce a polariton condensate at the bottom of the trap. In this paper, we discuss the implications of polariton traps, and how the concept can explain many experimental results [2-10]. Such a framework is helpful in understanding recent reports of nonlinear luminescence [11-13] and the suggested 'boser' [14].

\section{Pair scattering}

On a 2D quadratic dispersion relation such as that for the excitons or cavity photons, pair scattering near $k=0$ is not able to conserve energy and momentum. Thus exciton cooling is primarily via the emission of optic and acoustic phonons. However, the distortion of the dispersion relation produced by the polariton trap, switches on these previously forbidden scattering events, and pairs of excitons or polaritons can now 


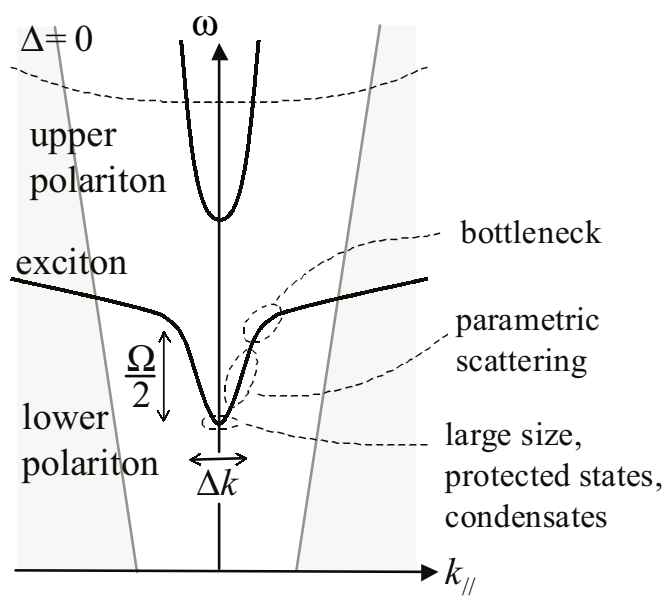

Fig. 1. In-plane dispersion relation of upper and lower polaritons showing the trap at small in-plane $k$. New effects arise from the sides, bottom and neck of the trap. The shaded region is not accessible from outside the sample.

interact via Coulomb dipole-dipole coupling. Recently, we demonstrated that if a pulse of polaritons is injected at the point of inflection on the side of the polariton trap (the so-called 'magic angle'), they can pair scatter to yield a polariton at the bottom of the trap (termed the 'signal') and a polariton that carries off the excess energy and momentum (the 'idler') [2]. In common with the formalism of nonlinear optics, this is known as a parametric scattering event. What is clear from our experiments is that this process can be stimulated, so that the scattering rate is increased by occupation of the final state. This relies on the bosonic properties of the polaritons, which are composite bosons, and only occurs at densities low enough for fermionic carrier interactions to be unimportant.

Parametric scattering is so strong (with gains $>10^{6} \mathrm{~cm}^{-2}$ ) that it dominates the $\mathrm{CW}$ emission properties of the semiconductor microcavity when pumped at the magic angle of $16.5^{\circ}$. As the pump power increases, the emission at $k=0$ increases exponentially, passing through a threshold around $40 \mathrm{~mW}$, and further increasing linearly $[3,4]$. The optical beam emerging at normal incidence is partnered by an idler emission at $35^{\circ}$ with the same power dependence, which is however much weaker. This microoptical parametric oscillator ( $\mu \mathrm{OPO}$ ) is a factor of 10,000 shorter than previous OPOs because of the enormous gain length, and is the dispersion engineered equiv- alent to vertical-cavity surface emitting lasers (VCSELs). Whereas lasing in VCSELs occurs at high $\mathrm{e}-\mathrm{h}$ densities so that excitons are screened out, oscillation in the $\mu \mathrm{OPO}$ is still governed by exciton-polariton emission.

Another effect of the strong parametric interaction is to shift and distort the dispersion relation on which the polaritons sit. The parametric interaction possesses both real and imaginary components of roughly equal magnitude. While the imaginary component can be negative, providing strong gains $(>1000)$, the real component produces an energy blue shift of the polariton dispersion [5]. Thus as the scattering switches on, the whole dispersion relation rigidly moves up in energy (by roughly the polariton line width). This implies that the pump beam needs to be tuned to higher energy to inject more polaritons in the cavity, producing a linear polariton energy shift with pump power that saturates depending on the line width and tuning of the pump [6]. Occupation of the polariton dispersion by a macroscopic population at a particular in-plane $k$ (as in the $\mu$ OPO above threshold) results in turning on new parametric scattering processes which further distort the dispersion relation. For the case when signal and idler polariton populations are produced (at occupations $N \sim 10^{4}$ ) in the initial scattering process, the strong occupations at pump, signal and idler wave vectors provide the possibility of further parametric scatterings, and these further distort the polariton dispersion relation. The key to understand this unusual behaviour is the anti-Hermitian or anomalous coupling produced by pair scattering [7]. Rather than the conventional anti-crossing or level repulsion produced by mixing exciton and photon states, the parametric coupling has a negative sign that produces a level attraction and brings the unmixed polariton energies together. This is illustrated in Fig. 2 in which polaritons are present at two general positions on the polariton dispersion, $k_{1,2}$. Further allowed parametric interactions can be found by rotating the polariton dispersion (dashed) by $180^{\circ}$ about the point $\left(^{*}\right)$ midway between $\left(\omega_{1}, k_{1}\right)$ and $\left(\omega_{2}, k_{2}\right)$-this produces a map (dotted) of all the states to which a pair of polaritons from $k_{1,2}$ can scatter into (Fig. 2a). However, the anomalous coupling also modifies the energies of these initial and final polariton states which are coupled together (dashed, dotted), producing a new dispersion relation (Fig. 2b, solid) onto which the 


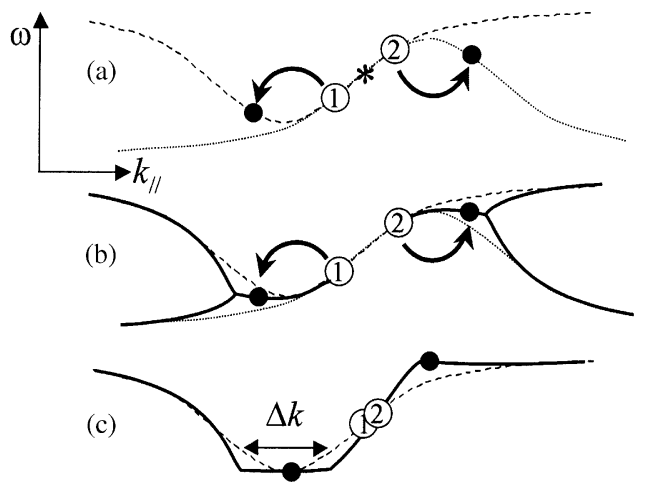

Fig. 2. (a) Polariton dispersion (dashed) macroscopically occupied at two points $(1,2)$ together with the set of points for which parametric scattering is energy-momentum conserved (dotted). This can be found by rotating the dispersion about the midpoint of the polariton pair involved $\left({ }^{*}\right)$. (b) Dispersion renormalized by parametric coupling, which attracts initial states that are close enough in energy. (c) Typical dispersion in the parametric regime, with flattened bottom and straight sides of the now asymmetrical polariton trap.

polaritons scatter. The extremely strong parametric coupling of polaritons on the lower branch produces a highly nonlinear system in which occupation at any wave vector initiates a cascade that not only scatters the polaritons, but also distorts their dispersion relation. The tendency of this anomalous coupling is to produce straight sections of polariton dispersion around occupied wave vectors, separated by sharp kinks. For the present case in which a strong signal polariton density is produced at $k=0$, together with an idler at $2 k_{\mathrm{p}}$, the new dispersion has a much flatter bottom to the polariton trap (Fig. 2c), as observed in many experiments [8]. In addition a negative effective mass is induced at the idler wave vector, as recently observed [7], which exemplifies the degree of control of polaritons possible in such a nonlinear system. This model predicts that strong occupation at the bottom of the polariton trap flattens out the dispersion in $k$-space, producing smaller polariton wave functions in real space. Note, however, that the lateral coherence length of the polariton is $\sim 100 \mu \mathrm{m}$, three orders of magnitude larger than that of the underlying excitons, due to wave function mixing with the cavity photons. By solving the parametric equations in Ref. [7], the model predicts that the angular width of this $k=0$ polariton increases and is set by the balance between that the confining potential energy and the parametric coupling, $V$, and is given by

$\Delta k\left(N_{0}\right)=\Delta k_{0}\left[1+\left(\frac{V N_{0}}{\gamma}\right)^{2}\right]^{1 / 4}$,

where $N_{0}$ is the polariton occupation of the $k=0$ state, $\gamma$ is the cavity line width, and $V$ is the Coulomb interaction strength $\sim 0.1 \mu \mathrm{eV} /$ polariton [7]. The decreasing real-space diameter of the $k \sim 0$ polariton implies that within the pumped area of the microcavity sample, the signal emission can break up into independent regions, or form patterns of spatial solitons. Such complex spatial behaviour has indeed been seen in far-field emission in high-Q semiconductor microcavities [8].

\section{Relaxation bottleneck}

When the microcavity is excited non-resonantly, an exciton population forms near the bottom of the exciton dispersion. However for excitons to scatter down into the trap requires that they lose a significant amount of energy, in our case equal to $\Omega / 2=4 \mathrm{meV}$. Excitons which have small in-plane momentum $k$ cannot emit optic or acoustic phonons to achieve this, and so are trapped above the neck of the polariton trap at low densities. The route that excitons take to drop into the polariton trap has been a source of controversy over recent years, with phonons, disorder, and exciton-exciton scattering processes all suggested. By imaging and spectrally resolving the emission from the microcavity we can show that exciton pair scattering, dominates the relaxation [9]. When a low-density cold exciton gas is excited with a CW non-resonant pump laser, emission near the bottleneck is seen emerging in an angular ring, with an energy just below the bare exciton energy. As the exciton density is increased, the angular radius of this ring shrinks, with the emission energy following the lower branch dispersion down into the trap. This can be explained only by an exciton-exciton pair scattering process in which to fall deepest into the polariton trap, two excitons with maximum kinetic energy, $E_{\mathrm{m}}=\hbar^{2} k_{\mathrm{m}}^{2} / 2 m$, mutually scatter to send one of them into the trap while the other takes up the extra energy and momentum. It is easy to show that the energy that can be removed is 
(a)
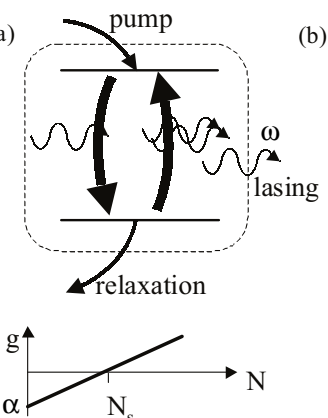

(b)
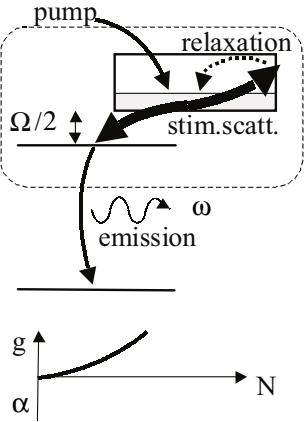

Fig. 3. Laser action on (a) a conventional two-level transition, and (b) on the pair scattering of a polariton trap. In each case the emission is at $\omega$, the thick lines show the stimulated transitions, while the dotted box contains the amplifier system. In (b) the pair scattering sends excitons into the trap while the dotted line shows the relaxation of higher energy excitons back to the thermalized distribution, which prevents re-ionization of polaritons from inside the trap (thermal lock). The pump dependence of the gain is shown below.

$2 E_{\mathrm{m}}$ due to the quadratic dispersion relation, scattering polaritons below the exciton energy by an amount $\Delta E_{\max }=\left(\hbar^{2} / 2 m\right)\left[\left(2 k_{\mathrm{m}}\right)^{2}-2 k_{\mathrm{m}}^{2}\right]$. This result is experimentally confirmed by the predicted increase of the magnitude of energy relaxation into the trap $\Delta E(T)$, when increasing the temperature, which increases the occupation of excitons at higher in-plane $k$. In complete contrast to behaviour in VCSELs, increasing the temperatures (at least initially) improves the relaxation rate. Such exciton pair scattering cannot occur on the quadratic exciton dispersion near $k \sim 0$ and is another result of the distortion of the polariton dispersion relation due to strong coupling of light and matter.

The relaxation of excitons into the polariton trap by pair scattering leads to a new form of laser emission. Most lasers are based on two-level transitions which can re-absorb photons once they have been emitted (Fig. 3a). The constraint of the Einstein $A$ and $B$ coefficients insists that to have gain requires the inversion of the transition. In a pair-scattering laser based on semiconductor microcavities it is possible to have gain without this inversion condition, because the stimulation and emission processes are separated. Excitons (either injected optically or electrically into the quantum wells) can be stimulated to form polariton inside the trap. These polaritons then decay into photons emerging from the microcavity, and any re-absorption is impossible since it requires the

ionization of polaritons out of the trap, which needs much higher energy excitons (Fig. 3b). Although the scattering-in process initially creates such high-energy excitons, they rapidly re-thermalize, and occupation of these high- $k$ states is very small. Gain thus builds up once the pair scattering rate into the polariton trap exceeds the cavity emission rate, producing a macroscopic polariton population at the bottom of the trap. The process is even more effective if the microcavity is doped with electrons, which provide an even stronger pair scattering with excitons. We predict that such n-doped microcavities can display extremely low thresholds with very fast switch-on times [10]. This is a new sort of laser based on strong-coupling, stimulated pair scattering, and fast thermalization of a reservoir, thus removing the need for inversion. It thus operates in a very different regime to VCSELs.

\section{Condensation}

When a microcavity is $\mathrm{CW}$ pumped either in the $\mu \mathrm{OPO}$ condition (Section 1), or non-resonantly with excitons which pair scatter into the trap (Section 2), the emission at $k=0$ builds up. The $k=0$ state that emits light is a polariton with a significant content of both photon and exciton (depending on the detuning and Rabi splitting), and is macroscopically coherent so that all quasiparticles have the same phase. This phase has no connection with that of the pump laser but spontaneously emerges from the vacuum noise, a form of spontaneous symmetry breaking. The state that forms can thus be considered a condensate, although this is a non-equilibrium state since the component polaritons decay from the microcavity rapidly and need to be replaced by stimulated scattering from the reservoir (Fig. 4a). The line width of the polariton condensate is measured interferometrically and found to be below $500 \mathrm{MHz}$ (Fig. 4b), indicating a collective phase coherence time $>1 \mathrm{~ns}$, three orders of magnitude longer than that of the excitons at this density. The bottom of the polariton trap is lower in energy than all other states in the semiconductor and this protects the polaritons from phase scattering processes. In this way once again the engineered polariton dispersion is able to generate new properties for quasiparticles. This condensate differs in a number of ways from a BEC: it is coherently pumped though 


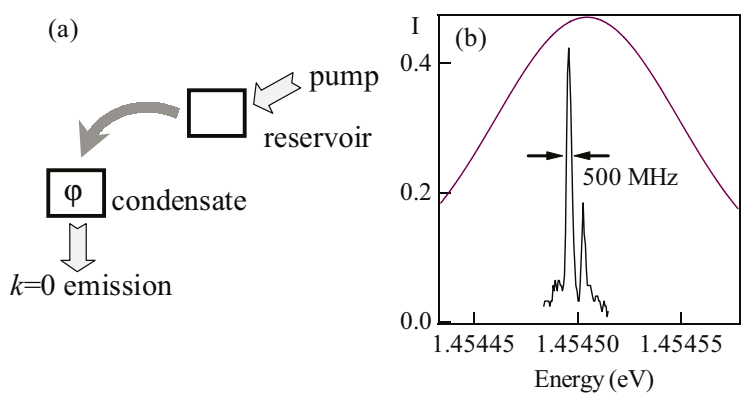

Fig. 4. (a) Schematic of the non-equilibrium polariton condensate which emits at the cavity decay rate, and thus needs to be continually pumped from an incoherent reservoir. (b) Measured polariton emission spectrum using a spectrometer (thin line) and a plane-plane Fabry-Perot interferometer (thick line) of spectral resolutions $0.1 \mathrm{meV}$ and $4 \mu \mathrm{eV}$, respectively.

this is not necessary for its operation, it is manifestly non-equilibrium so it is hard to define a chemical potential, and it exists in the solid state. On the other hand, it shares many features such as: a macroscopic phase which can be used for making interferometric devices such as Josephson junctions, a condensate energy gap in the density of states and a Boglubov-like excitation spectrum, and a spin degree of freedom. The condensate differs from a normal laser in that the photon emission is from a state which is half-light and half-matter, and it is the scattering rather than the emission which is stimulated. In some ways, these condensates form a bridge between the concepts of a laser and of a superfluid, with the disadvantage that they decay rapidly but the advantage that their coherent emission makes them easy to study.

\section{Conclusions}

We have shown that polariton traps are a generic concept for describing much of the polariton dynamics in semiconductor microcavities. The sides of the trap switch on the process of parametric scattering, the neck of the trap controls polariton relaxation and can produce a new type of laser action, and the bottom of the trap is an extremely stable semiconductor excitation that can protect against phase scattering. At the present time, a number of groups are working on room temperature operation (the trap depth must exceed the thermal energy, $\Omega>2 k_{\mathrm{B}} T$ ) either by heterostructure engineering or new material systems. The flexibility and utility of polaritons can potentially be utilized in devices such as interferometers, and ultrafast optical amplifiers.

\section{Acknowledgements}

We thank Cristiano Ciuti, Diego Porras, Alexey Kavokin, Guillaume Malpeuch for much stimulating input and discussions.

\section{References}

[1] R. Houdre, et al., Phys. Rev. Lett. 73 (1994) 2043.

[2] P.G. Savvidis, et al., Phys. Rev. Lett. 84 (2000) 1547.

[3] J.J. Baumberg, et al., Phys. Rev. B 62 (2000) R16247.

[4] R.M. Stevenson, et al., Phys. Rev. Lett. 85 (2000) 3680.

[5] C. Ciuti, P. Schwendimann, A. Quattropani, Phys. Rev. B 62 (2000) R4825.

[6] P.G. Savvidis, et al., Phys. Rev. B 62 (2000) R13278.

[7] P.G. Savvidis, et al., Phys. Rev. B 64 (2001) 75311.

[8] R. Houdre, C. Weisbuch, R.P. Stanley, U. Oesterle, M. Ilegems, Phys. Rev. Lett. 85 (2000) 2793.

[9] P.G. Savvidis, et al., Phys. Rev. B (2002) in press.

[10] G. Malpeuch, A. Kavokin, A. diCarlo, J.J. Baumberg, Phys. Rev. B (2002) in press.

[11] P. Senellart, et al., Phys. Rev. Lett. 82 (1999) 1233.

[12] Le Si Dang, et al., Phys. Rev. Lett. 81 (1998) 3920.

[13] S. Pau, et al., Phys. Rev A 54 (1996) R1789.

[14] A. Immamoglu, et al., Phys. Rev. A 53 (1996) 4250 